Cahiers $d u$ MONDE RUSSE

\section{Cahiers du monde russe}

Russie - Empire russe - Union soviétique et États indépendants

$44 / 4 \mid 2003$

Varia

\title{
Natalia Borisovna Lebina, Aleksandr Nikolaevič Čistikov, Obyvatel' i reformy
}

\author{
Larissa Zakharova
}

\section{(2) OpenEdition}

Journals

Édition électronique

URL : https://journals.openedition.org/monderusse/4129

DOI : 10.4000/monderusse.4129

ISSN : $1777-5388$

Éditeur

Éditions de l'EHESS

Édition imprimée

Date de publication : 1 octobre 2003

Pagination : 772-775

ISBN : 2-7132-1833-0

ISSN : $1252-6576$

Référence électronique

Larissa Zakharova, "Natalia Borisovna Lebina, Aleksandr Nikolaevič Čistikov, Obyvatel" i reformy », Cahiers du monde russe [En ligne], 44/4 | 2003, mis en ligne le 19 juin 2009, consulté le 04 septembre 2022. URL : http://journals.openedition.org/monderusse/4129 ; DOI : https://doi.org/10.4000/ monderusse. 4129

Ce document a été généré automatiquement le 4 septembre 2022.

Tous droits réservés 


\title{
Natalia Borisovna Lebina, Aleksandr Nikolaevič Čistikov, Obyvatel' i reformy
}

\author{
Larissa Zakharova
}

\section{RÉFÉRENCE}

Natalia Borisovna LEBINA, Aleksandr Nikolaevič ČISTIKOV, Obyvatel' i reformy.

Kartiny povsednevnoj žizni gorožan v gody NEPa i hruščevskogo desjatiletija.

Saint-Pétersbourg, « Dmitri Bulanin », 2003, 340 p.

1 Le livre de Natalia B. Lebina et Aleksandr N. Čistikov, Habitant et réformes. Images de la vie quotidienne des citadins dans les années de la NEP et la décennie khrouchtchévienne, est classé dans le genre des "essais historiques" et destiné à un large public. Les auteurs s'interrogent sur l'impact des réformes sur le train de vie des gens ordinaires à Leningrad au cours des années 1920 d'une part, et 1950-1960 de l'autre. Ils essaient de définir le décalage temporel entre la proclamation d'une réforme et les transformations réelles qu'elle suscite dans le mode de vie, notamment dans les sphères du logement, des pratiques vestimentaires, de la santé (alimentation, hygiène, sport), des pratiques sociales marginales (alcoolisme, toxicomanie, hooliganisme, prostitution, criminalité), et enfin des loisirs. Et ils tentent, par le biais de cette analyse, d'« extraire le code symbolique et sémiotique de chaque période», tel qu'il s'exprime au travers des pratiques corporelles.

2 La structure du livre repose sur ces deux ensembles chronologiques. L'ouvrage est constitué de deux parties dans lesquelles les aspects mentionnés de la quotidienneté sont présentés dans des chapitres séparés. Chaque partie est précédée d'un texte introductif qui brosse un tableau de la structure urbaine de Leningrad à travers les changements dans le paysage spatial et la toponymie, l'économie municipale et la caractérisation des différents types sociaux qui parcourent la ville. 
3 L'analyse de la vie de tous les jours sous la NEP est plus approfondie que celle des années khrouchtchéviennes, car la première partie synthétise les idées exposées dans le précédent ouvrage de N.B. Lebina ${ }^{1}$. Seul le chapitre sur l'alimentation et le système sanitaire est issu d'une étude récente, effectuée à partir de mémoires, de journaux intimes et de la presse des années 1920. Les conditions de vie pendant la période du communisme de guerre ont fait apparaitre des pratiques spécifiques dans l'obtention de la nourriture : pajkolovstvo (ou ruse pour obtenir des rations), alimentation obligatoire dans les cantines municipales. Cet état de choses ne change pas immédiatement avec le passage à la NEP. C'est à partir de l'automne 1921 que le menu des habitants de Petrograd s'améliore qualitativement et quantitativement. Les marchés, les coopératives, les restaurants (avec leurs traditions culinaires d'avant la révolution) et les magasins d'État entrent en concurrence pour attirer le consommateur. Le lien qu'établissent les auteurs entre les pratiques corporelles des citadins (alimentation, soins médicaux, hygiène, sport) et l'espace urbain (lieux fréquentés tels que bars, restaurants, pharmacies, sanatoriums, stades, etc.) rend cette étude pertinente.

4 En ce qui concerne le logement, les auteurs ont mis l'accent sur la redistribution des appartements dans le centre-ville qui reflète bien le caractère autoritaire de cette mesure, voulue comme l'attribution d'un privilège accordé aux classes auparavant dominées et perçue sans enthousiasme par les «déplacés». On observe aussi d'autres expérimentations dans le domaine du logement : concentration des dirigeants dans des lieux prestigieux (l'Institut Smolny, les meilleurs hôtels et les plus beaux immeubles), apparition de communes de jeunes, entassement de nouveaux locataires dans les appartements «bourgeois ». Le climat de la NEP devient perceptible dans l'habitat au milieu de l'année 1922 : la renaissance d'un marché de l'immobilier et l'échelle des prix des loyers témoignent d'une différenciation sociale renforcée.

On observe la même évolution dans les pratiques vestimentaires. La redistribution centralisée de vêtements continue jusqu'en automne 1922. Ensuite, la pénurie vestimentaire touche la majorité des citadins et même les ouvriers, alors que cette catégorie avait régulièrement bénéficié de distribution d'habits pendant le communisme de guerre. C'est à partir de 1925 que l'« atelier» (lieu où l'on peut se faire faire des vêtements sur mesure, auparavant réservé aux fonctionnaires haut placés) devient accessible au citadin ordinaire. Le retour de cette pratique prérévolutionnaire a pour conséquence la renaissance de la mode. Les vêtements redeviennent des signes de stratification sociale.

6 Cette dynamique de la NEP entraîne des effets indésirables : falsification de la monnaie, hausse de la criminalité, marginalisation de la culture urbaine (du fait du déplacement des ouvriers vers le centre-ville), retour de la prostitution, accès facilité à l'alcool et à la drogue, développement des jeux de hasard. Parmi les loisirs socialement tolérés, les auteurs énumèrent les visites chez des amis, les promenades en ville et à la campagne, les séjours à la datcha, la participation à des festivités populaires, le cinéma, le théâtre et la lecture.

7 L'influence du discours officiel sur le mode de vie n'est pas moins important pendant le «dégel ». Les auteurs affirment que la sphère la plus touchée par les réformes khrouchtchéviennes a été l'habitat, mais il faut rappeler que la campagne prônant l'attribution d'un appartement individuel à chaque famille remonte aux grands chantiers de l'après-guerre. La "déstalinisation » dans ce domaine a commencé à Leningrad à la veille $d u X X^{e}$ Congrès du PCUS. On peut observer une évolution qui va d'un goût 
« bourgeois » dans le mobilier (selon les auteurs) et d'un style Empire dans l'architecture stalinienne, à une plus grande simplicité et une forme de standardisation dans la décoration intérieure et l'architecture des années 1950-1960. Cette tendance obéit à un concept de rationalité et de modernité. Le minimalisme devient une règle incontournable : les créateurs de mobilier recherchent les formes les plus économiques et s'efforcent de gagner de la place en agrandissant l'espace grâce à un effet de trompel'œil. La modernisation de l'espace habitable est associée à l'apparition des appareils ménagers et à celle des téléviseurs qui ouvrent une nouvelle ère dans la communication. Dans toute cette partie de l'ouvrage consacrée à l'habitat, on peut regretter que les auteurs traitent davantage des projets de construction que des pratiques réelles des citadins.

8 Du fait d'une lecture trop peu critique de la littérature soviétique officielle, l'étude de l'alimentation des habitants de Leningrad pendant le «dégel» présente un tableau incontestablement trop favorable de l'amélioration progressive de la nutrition au cours des années 1950. La famine de l'après-guerre n'y est même pas mentionnée. Le déficit alimentaire de la deuxième moitié de la période est expliqué par un regain de pression sur les agriculteurs auxquels sont fixés des objectifs de production exagérés en vue de rattraper les États-Unis. Les changements qualitatifs dans l'alimentation, engendrés par les réformes, sont associés aux nouvelles formes de commerce (self-service), à l'automatisation de l'alimentation collective (obščestvennoe pitanie) et à l'apparition de nouveaux aliments (notamment du maïs).

9 Les normes d'hygiène restent liées à l'accès aux commodités sanitaires (salles de bain, douches, bains publics). La propagande en faveur d'un mode de vie sain a des effets visibles dans les sports pratiqués par les citadins. Mais malgré le discours officiel sur l'émergence de l'homme nouveau-citoyen de la société communiste, la consommation d'alcool n'est pas combattue avant 1959, alors que ce fléau touche toutes les couches sociales. De 1959 à 1963, des mesures restrictives sont prises, notamment une réduction des budgets municipaux qui entraîne, par contrecoup, une hausse de la production d'alcool domestique.

Le discours officiel khrouchtchévien crée des stéréotypes de la marginalité. Sont ainsi stigmatisés les pratiques religieuses, la dissidence, la spéculation, la subculture des zazous (stiljagi) et le jazz, la lecture de Remarque et de Hemingway, etc. À l'opposé, les volontaires (družinniki) appelés à lutter contre la criminalité incarnent un type social positif de cette époque.

11 Parmi les nouvelles formes de loisirs caractéristiques des années 1950-1960, les auteurs mettent l'accent sur le tourisme et les «clubs de l'amitié internationale ». Les loisirs «traditionnels» (promenades, rassemblements dans les lieux publics, discussions, lecture, cinéma, concerts) sont aussi étudiés.

12 Dans l'étude des pratiques vestimentaires des années 1950-1960, on pourra regretter un style trop descriptif, qui est d'ailleurs un écueil classique dans l'histoire de la vie quotidienne, ainsi que l'insuffisance des données quantitatives. Les auteurs insistent sur la diffusion des modes occidentales à Leningrad, mais les canaux de transfert ne sont pas retracés. L'évolution de la mode à Leningrad n'est pas mise en parallèle avec celle de la mode occidentale. Ainsi les tendances « de luxe » incarnées par la maison Christian Dior sont associées par les auteurs au seul style «new look». C'est pourquoi le moment où ce style devient démodé est présenté de manière erronée dans l'ouvrage comme le refus des modélistes de Leningrad de suivre la mode Dior. Le discours conceptualisant la mode 
«socialiste » - fonctionnelle et utilitaire - y est identifié aux principes de Chanel en matière de modélisme, mais ni la double logique de ce discours, ni les preuves concrètes de l'adaptation de la mode Chanel dans la mode soviétique ne sont explicitées.

13 Les quelques imperfections signalées plus haut peuvent être imputées au caractère fragmentaire des sources et sont pardonnables pour ce genre d'ouvrage. Il reste que le livre séduit par sa problématique neuve, ses cadres d'analyse et une appréciation fine de l'atmosphère des deux époques étudiées.

\section{NOTES}

1. N. B. Lebina, Povsednevnaja žizn' sovetskogo goroda: normy $i$ anomalii. 1920-1930 gody, SaintPétersbourg, « Kikimora », « Neva », «Letnij sad », 1999, 317 p. 